\title{
The Expedited International Standards for Neurological Classification of Spinal Cord Injury (E-ISNCSCI)
}

\author{
Stephen P. Burns ${ }^{1,2} \cdot$ Keith E. Tansey ${ }^{3,4,5}$
}

Received: 27 March 2020 / Accepted: 27 March 2020 / Published online: 6 April 2020

(c) International Spinal Cord Society 2020

The International Standards for Neurological Classification of Spinal Cord Injury (ISNCSCI) [1] is a comprehensive, thorough, and exhaustive clinical examination of segmental somatic sensory and motor function used to derive standardized neurological classifications. Many examiners and examinees would also describe the ISNCSCI as exhausting! The exam requires sensory testing of 28 dermatomes for light touch and sharp/dull discrimination bilaterally, manual muscle testing for ten key limb muscles bilaterally, plus an anorectal exam for sensation and motor function. In sum, this detailed exam of testable segments includes 134 items. At times, completion and recording of the exam can take in excess of $45 \mathrm{~min}$, even for an experienced examiner.

Due to this time requirement, SCI care providers and other clinicians have been performing nonstandardized, abbreviated exams when the full exam and standardized classification is not required. Also, in circumstances when testing of the S4-5 dermatomes and an anorectal exam cannot be performed, there has been no alternative for assigning a neurologic classification. This limits community-based research projects in which neurologic status is merely being described and is not a study outcome.

Stephen P. Burns

spburns@uw.edu

1 Spinal Cord Injury Service, VA Puget Sound Health Care System, Seattle, WA, USA

2 Department of Rehabilitation Medicine, University of Washington School of Medicine, Seattle, WA, USA

3 Departments of Neurosurgery and Neurobiology, University of Mississippi Medical Center, Jackson, MS, USA

4 Center for Neuroscience and Neurological Recovery, Methodist Rehabilitation Center, Jackson, MS, USA

5 Spinal Cord Injury Clinic, Jackson VA Medical Center, Jackson, MS, USA
Clinicians and researchers have thus asked the International Standards Committee of the American Spinal Injury Association (ASIA) to develop a rigorous, shortened exam option that could be used in appropriate clinical or research applications. This could potentially include screening exams, daily exams, or periodic follow-up exams in those without new neurological complaints or changes in functional status.

In February 2020, after 3 years of development, the Expedited ISNCSCI (E-ISNCSCI) protocol was posted on the ASIA website [2]. The E-ISNCSCI protocol aims to define the neurological level of injury (NLI) and ASIA impairment scale (AIS) with the fewest exam items. This is achieved in part by omitting exam items that do not influence NLI and AIS determination, and through options that allow assumptions of some exam findings based on other findings. Depending on the severity of injury, this can allow omission of $75 \%$ or more of the 134 items in the full ISNCSCI exam. There are options to substitute S1 sensory and motor findings for the anorectal exam and S4-5 dermatomes if these cannot be performed [3] or omit upper limb testing when the likelihood of upper limb weakness is low. Rates for errors introduced by shortcut options have been determined using large databases of full ISNCSCI exams, and these analyses guided E-ISNCSCI development. For example, the protocol specifies a cutoff NLI (T10) below which there is reduced correlation between S1 findings and the anorectal exam findings, and for such cases the S1 substitution option should not be used. It is recognized that the two protocols may produce conflicting AIS classifications, but not likely NLI determinations, in certain cases. Because the E-ISNCSCI will not always produce a classification identical to the full ISNCSCI, clinicians and researchers must note if the E-ISNCSCI was used to derive a classification. In early presentations of this new exam version at ASIA annual scientific meetings, there has been good reception and appreciation for a relatively precise, shortened exam. 
The primary concern of the International Standards Committee is that the E-ISNCSCI could be used inappropriately in circumstances that require the full ISNCSCI. The protocol document includes guidance on some circumstances that require the full ISNCSCI (“....in the acute setting, on admission and discharge from rehabilitation, in patients with chronic SCI and suspected neurological changes, or for protocol-mandated clinical trials.") and a warning that the EISNCSCI should only be performed by experienced examiners who are knowledgeable in the use of the full ISNCSCI exam [2]. Available training on the E-ISNCSCI is currently limited to the referenced protocol document and four classification case examples posted on the ASIA website. For the full ISNCSCI, the exam process can be summed up as "test all the dermatomes, test all the key muscles (and non-key muscles as needed), and perform the anorectal exam," whereas the sequence of examination for E-ISNCSCI will vary depending on what the initial part of the exam shows. Thus, comprehensive instruction on performing the E-ISNCSCI must include the decision points during the examination. The examination technique and individual examination components are identical to the full ISNCSCI, and the classification rules essentially follow the full ISNCSCI.
The International Standards Committee anticipates revisions to the E-ISNCSCI protocol may be needed to clarify the instructions and facilitate teaching. Clinicians and researchers are encouraged to participate by submitting feedback to the International Standards Committee per the instructions on the ASIA website.

Publisher's note Springer Nature remains neutral with regard to jurisdictional claims in published maps and institutional affiliations.

\section{References}

1. International Standards for Neurological Classification of Spinal Cord Injury worksheet. 2019. https://asia-spinalinjury.org/interna tional-standards-neurological-classification-sci-isncsci-worksheet/. Accessed 2 Mar 2020.

2. Expedited ASIA ISNCSCI Exam (E-ISNCSCI) Version 1 (February 2020). 2020. https://asia-spinalinjury.org/expedited-isncsciexam/. Accessed 23 Mar 2020.

3. Zariffa J, Kramer JL, Jones LA, Lammertse DP, Curt A, Steeves JD, European Multicenter Study about Spinal Cord Injury Study Group. Sacral sparing in SCI: beyond the S4-S5 and anorectal examination. Spine J. 2012;12:389-400. 